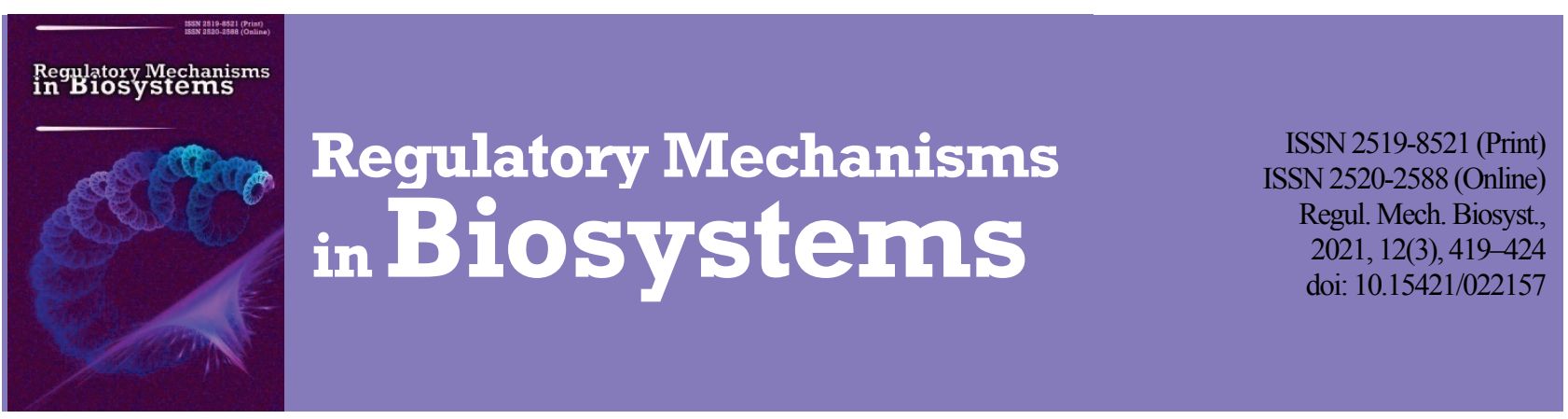

\title{
The effect of Corvitin on the content of bile acids in the liver of rats under conditions of chronic social stress
}

\author{
A. M. Liashevych*, I. S. Lupaina*, T. L. Davydovska**, O. V. Tsymbalyuk**, Y. R. Oksentiuk*, M. Y. Makarchuk** \\ *Zhytomyr Ivan Franko State University, Zhytomyr, Ukraine \\ **Taras Shevchenko National University of Kyiv, Kyiv, Ukraine
}

Article info

Received 09.06.2021 Received in revised form 13.07.2021

Accepted 14.07.2021

Zhytomyr Tan Franko State University,

Velyka Berdychivska st. 40, Zhytomyr, 10002, Ukraine Tel.: +38-063-050-22-30. E-mail:

lam88leona2@gmail.com

Taras Shevchenko

National University of Kyiv Glushkova st. 2,

Kyiv, 03022, Ukraine.

Tel.: + 38-044-239-33-33.

E-mail:

office.chief@univ.net.ua

\begin{abstract}
Liashevych, A. M., Lupaina, I. S., Davydovska, T. L., Tsymbalyuk, O. V., Oksentiuk, Y. R., \& Makarchuk, M. Y. (2021). The effect of Corvitin on the content of bile acids in the liver of rats under conditions of chronic social stress. Regulatory Mechanisms in Biosystems, 12(3), 419-424. doi:10.15421/022157
\end{abstract}

The article looks at recent research dealing with changes in the bile acid composition of the bile of outbred male rats under chronic social stress (social defeat in daily male confrontations, 14 days) when administered Corvitin ( $1 \mathrm{mg} / \mathrm{kg}$, intragastrically, 7 days). Chronic social stress was created by daily agonistic interactions between animals. The main fractions of conjugated bile acids - taurocholic, taurohenodeoxycholic and taurodeoxycholic, glycocholic, glycochenodeoxycholic and glycodeoxycholic and free ones - cholic, chenodeoxycholic and deoxycholic were determined by the method of thin layer chromatography of bile. The conjugation index (ratio of the sum of conjugated cholates to the sum of free ones) and hydroxylation (ratio of the sum of trihydroxycholanic bile acids to the sum of dihydroxycholanic ones) of bile acids were calculated. The research showed that in the conditions of experimental social stress, Corvitin enhances the conjugation of bile acids with taurine and glycine, i.e. stimulates detoxification processes in hepatocytes. In the conditions of chronic social stress in male rats, the processes that had provided the flow of glycoconjugates of bile acids from hepatocytes to the bile ducts were further suppressed. The concentrations of glycocholic acid and glycochenodeoxycholic and glycodeoxycholic acids in the bile of male intruders were lower than the control values. But, as seen in the experiment, the use of Corvitin normalized these indicators. The experiment showed that in the conditions of chronic social stress, the content of cholic acid in the bile of intruder rats decreased, and when correcting the pathological condition using Corvitin, it reached the control values. The use of Corvitin simultaneously with the simulation of experimental social stress normalized the biliary secretory function of the liver, indicating the high potential of using Corvitin as a corrective factor in chronic social stress. Correction of stress-induced pathologies of liver bile-secretory function by Corvitin requires further thorough experimental studies.

Keywords: cholates; bile secretion rate; bile acid conjugation; bile acid hydroxylation; social defeat model.

\section{Introduction}

The liver is the largest gland in the digestive system. It is the key organ in the intermediate metabolism and its role in the adaptive response is exceptional, because the liver performs its many functions throughout the life of an individual. The study of the peculiarities of the digestive system functioning under stress is currently quite relevant. Disorders in the system of neurohumoral regulation play a significant role in the mechanisms of decompensation of the body's adaptive capabilities under stress. Corticosteroid hormones are important in the humoral component of this system, which control both the metabolic supply of organs and the state of tissue regulatory systems that form the body's protective reactions (Boyer, 2013; Juza \& Pauli, 2014).

Liver function is subject to complex neurohumoral regulation, and any stress-induced shifts in neural and humoral regulatory mechanisms have a direct or indirect effect on many functions of the liver (Boyer, 2013; Silvennoinen et al., 2015; Jensen et al., 2016). Enzymatic systems of hepatocytes provide the synthesis of cholesterol and the formation of bile acids, which are its only end metabolites excreted from the body and which have a wide range of physiological effects (necessary for the normal course of digestive processes involved in regulating a large number of intrahepatic and systemic processes (Zhou \& Hylemon, 2014; Pierre et al., 2016; Taoka et al., 2016; Chiang, 2017; Yan \& Lun-Gen, 2018; Lieshchova et al., 2018; Brygadyrenko et al., 2019).

One of the identified effects of chronic social stress is a significant change in the ratio of serum lipoproteins and blood lipid spectrum, choles- terol synthesis and receptor-mediated transport, fatty acid synthesis and oxidation and cholesterol and triglyceride content in liver cells and other lipid disorders (Chuang et al., 2010; Scott et al., 2012; Shkurashivska \& Ersteniuk, 2015). The key reactions of cholesterol metabolism take place in the liver. In particular, the enzymatic systems of hepatocytes provide the formation of bile acids from cholesterol, which are its only end metabolites and can be excreted from the body (Zhao \& Dahlman-Wright, 2010; Chiang, 2013; Vasheka et al., 2014). However, bile acids have a fairly broad range of physiological effects. These unique cholesterol metabolites perform a "nutritional" function and are compounds involved in the regulation of a large number of intrahepatic and systemic processes (Trauner et al., 2010; Fuchs et al., 2013; Zhou et al., 2014; Pierre et al., 2016; Maleszka et al., 2017).

It should be noted that a key factor in the development of pathological conditions associated with disorders of cholesterol and bile acids in the liver, and hence a highly specific marker of liver damage, are changes in physiological ratios of different fractions of bile acids in bile and blood plasma. Such pathological changes are associated not only with disorders of cholate synthesis in hepatocytes, but also with impairments in their transport, biotransformation, recirculation when the body is under stress (Silvennoinen et al., 2015). However, these aspects of the effects of social stress on the metabolic transformations of cholesterol and its derivatives cholates remain undeservedly little studied.

Research has confirmed that stress-induced liver pathology can have negative effects on the whole body, causing impaired immunological tolerance and immunodeficiency, disease of the cardiovascular (e.g. atheros- 
clerosis) and musculoskeletal system (such as osteodystrophy, osteomalacia) and affect other organs and systems (Juza \& Pauli, 2014; Giudetti et al., 2019; Yun-zi et al., 2019). In the conditions of stress, a complex of biochemical reactions is formed in the liver, which can cause the development of stress-induced mechanisms of hepatocyte damage (Chuang et al., 2010).

Development of universally effective and safe correctors of biliary secretion disorders is becoming more relevant. There is an urgent need for scientists to find drugs that would effectively and without limiting side effects correct blood cholesterol levels and metabolism in the liver. Substances that significantly affect liver function include bioflavonoids, including quercetin. Quercetin and its soluble form of Corvitin are important representatives of the flavonoid family and food antioxidants that have the most significant effect. Numerous pharmacological effects of quercetin include protection against diseases such as atherosclerosis, myocardial infarction and cerebrovascular pathology. Corvitin also increases blood flow in the liver, but its effect on exocrine liver function has not been fully studied. Despite various studies of quercetin as a corrective agent in various pathologies of the body's visceral systems, the effectiveness of its water-soluble form - Corvitin in stress-induced disorders of biliary secretion of the liver is little known and requires detailed experimental studies.

The purpose of our study was determined by the growing importance of stress-induced disorders of the hepatobiliary system, primarily caused by social stress, and the urgent need to find effective drugs to correct stress-induced liver pathologies.

\section{Material and methods}

The study of the effect of Corvitin on the liver biliary function under chronic social stress was performed on white outbred male laboratory rats weighing $220 \mathrm{~g}$, which were kept in an accredited vivarium of The Educational-Scientific Centre the Institute of Biology and Medicine of Taras Shevchenko National University of Kyiv according to the "Standard rules for the organization, equipment and maintenance of experimental biological clinics (vivariums)". The research on the animals was performed in compliance with the international principles of the European Convention for the Protection of Vertebrate Animals used for research and other scientific purposes. All animal experiments were performed in accordance with the Declaration of Helsinki (World Medical Assembly, 1964), the Declaration of Principles on Tolerance (28th session of UNESCO, 1995), the Universal Declaration on Bioethics and Human Rights (United Nations, 1997), the Convention on Human Rights and Biomedicine, adopted in 1997 in Oviedo (Spain) and signed by the Verkhovna Rada of Ukraine in 2002, Law of Ukraine № 3447 IV "On protection of animals from cruel treatment". The experimental research conducted during the dissertation work in no way contradicts the generally accepted bioethical norms and was carried out in compliance with the relevant international regulations concerning the conduct of experimental work and clinical research.

The studied animals were kept in a vivarium in the air temperature of 22-24 ${ }^{\circ} \mathrm{C}$ with a 14-hour light period of the day, fed according to the standard diet with free access to water. The scheme of the experiment included modeling chronic social stress, which was caused by repeated experience of social defeats in daily inter-male confrontations for 14 days. Animals were kept in plastic cages with a lattice iron top individually $(\mathrm{n}=9)$ or a harem (one male was kept together with 2-4 females). The lighting condition in the vivarium was natural. The animals received the standard diet (compound feed for laboratory rats; Vitamex Ukraine) and had free access to water and food. The marking of animals was performed by applying numbers on the tails with a permanent marker. From the first to the last day of the study, the body weight of experimental animals was monitored.

Nine rats weighing $220 \mathrm{~g}$ were used as intruders in the model of chronic social stress. The other nine animals, weighing $330 \mathrm{~g}$, kept with 2 to 4 females, served as resident animals in the model of chronic social stress. Subsequently, for 14 days, intruder rats $(n=9)$ were exposed to chronic social stress of the social defeat model. From the seventh to the fourteenth day of the stress procedure, male intruder rats received Corvitin intragastrically (PJSC SIC "Borshchahivskiy Chemical-Pharmaceutical Plant', Ukraine) in the dose of $1 \mathrm{mg} / \mathrm{kg}$ body weight (Vovkun et al., 2016). Animals in the control group weighing $220 \mathrm{~g}(\mathrm{n}=8)$ also received the standard diet and had free access to water, but were not exposed to chronic stress of the social defeat model.

The choice of the social stress model was based on the following criteria: the ability to acquire subordinate status and the duration of influence sufficient to form an appropriate behavioural stereotype. The model of social defeat meets the specified criteria. The model of developed chronic social stress was modified for mice in which stress was caused by repeated experiences of social defeats in daily inter-male confrontations (Kudryavtseva, 1991; Horid'ko et al., 2017). The procedure of social stress began with a five-day isolation of intruder rats (experimental animals) to eliminate previous social experience. Subsequently, these animals were kept individually throughout the experiment. For three days before the onset of stress, the resident rats were tested for agonistic behaviour by putting indifferent rats that were not a part of the experimental animals into their cages. Resident rats that did not show aggressive behaviour were excluded from the experiment.

Stress was caused by daily agonistic interactions between the animals. Females were taken out of the cells of domestic rats 10 min before the start of agonistic interactions. Each of 14 days, in a darkened room, in the second half of the day, an intruder was placed in a cage with resident animals for 10 minutes for agonistic interactions. After that, a transparent perforated partition was installed in the cage for 20 minutes, which allowed visual, smelling and auditory, but not physical contact of rats. The number of attacks by resident rats and their activity were controlled. At the same time, the behaviour of intruders while in the same cage with the domestic rats was monitored as well.

One day after the procedure of chronic stress, we studied the biliary secretion function in acute experiments in intruder rats that were administrated Corvitin intragastrically. Before the surgery to obtain hepatic secretion, the animals were subjected to food deprivation, but they had free access to water. Each animal was weighed and labeled prior to surgery. Sodium thiopental (Kyivmedpreparat JSC, Ukraine) in the dose of $60 \mathrm{mg} / \mathrm{kg}$ was used for anesthesia. After laparotomy, the bile duct was cannulated and after 30 minutes of stabilization, the animal was taken for the next 3 hours of acute experiment, 18 10-minute bile samples were taken. Every three portions of bile were collected to one eppendorf and the content of different cholates in each 30-minute bile sample was further analyzed. The mean volumetric rate of bile secretion was calculated as the volume of hepatic secretion produced by $1 \mathrm{~g}$ of the liver in $1 \mathrm{~min}$. The concentration of bile acids and lipids in 30-minute bile samples was determined using a thin-layer chromatographic technique developed in the Department of General Physiology of Petro Bohach Research Institute of Physiology of Institute of Biology and Medicine of Taras Shevchenko National University of Kyiv (Veselskyy, S. P., Lyashchenko, P. S., \& Luk'janenko, Y. A. (1991). Sposob opredelenyja zhelchnyyh kyslot v byologycheskyh zhydkostjah. Avtorskoe svydetelstvo 1624322). The applied method allowed us to determine the following fractions of bile acids in the bile of rats: taurocholic, taurohenodeoxycholic and taurodeoxycholic (in the mixture), glycocholic, glycochenodeoxycholic and glycodeoxycholic (in the mixture), and cholic, chenodeoxycholic and deoxycholic acids (in the mixture).

Conjugation indices (ratio of total conjugated cholates (sum of concentrations of taurocholic, taurohenodeoxycholic and taurodeoxycholic acids, glycocholic and glycochenodeoxycholic and glycodeoxycholic acids) were calculated by value of concentration of conjugated and free cholates to total free bile acids (total concentration of cholic, chenodeoxycholic and deoxycholic acids) and hydroxylation of bile acids (the ratio of total trioxycholanic acids (total concentration of taurocholic, glycocholic and cholic acids) to the total deoxycholic acids (total concentration of taurochenodeoxycholic and taurodeoxycholic, glycochenodeoxycholic and glycodeoxycholic, chenodeoxycholic and deoxycholic acids), bile acids) and the ratio of glyco- and tauroconjugates of bile acids.

The obtained results were statistically analyzed in Statistica 10 software (StatSoft Inc., USA, 2011). Statistical processing of the obtained results was performed using the ANOVA, the data in the tables are presented in the form of $\mathrm{x} \pm \mathrm{SD}$ (mean \pm standard deviation). The difference was considered significant at $\mathrm{P}<0.05$ (taking into account the Bonferroni correction). 


\section{Results}

Throughout the experiment, one day after the end of the experimental social stress procedure, no statistically significant differences in the volumetric rate of bile secretion were found compared with the control values (Fig. 1). The rate of bile secretion ranged $1.2 \pm 0.5 \mu \mathrm{L} / \mathrm{min} \bullet \mathrm{g}$ of liver. Thus, at the beginning of the experiment, the average volumetric rate of bile secretion decreased to $1.4 \pm 0.4 \mu \mathrm{L} / \mathrm{min} \bullet \mathrm{g}$ of liver, i.e. by $9.7 \%$ compared with control values $-1.6 \pm 0.3 \mu \mathrm{L} / \mathrm{min} \bullet \mathrm{g}$ of liver. And after 2.5 3.0 hours of acute experiment, the average volumetric velocity increased to $1.1 \pm 0.6 \mu \mathrm{L} / \mathrm{min} \bullet \mathrm{g}$ of liver, i.e. by $15.3 \%$ compared with the control group of animals (Fig. 1). No statistically significant differences in the mean volumetric rate of bile secretion were observed in male rats that received Corvitin in the conditions of chronic social stress compared to control values.

The rate of bile secretion ranged $1.1 \pm 0.4 \mu \mathrm{L} / \mathrm{min} \bullet \mathrm{g}$ of liver (Fig. 2). At the beginning of the experiment, i.e. for 10-30 minutes of the experiment, the mean volumetric rate of bile secretion decreased to $1.2 \pm 0.4$ $\mu \mathrm{L} /$ min॰g of liver, i.e. by $26.5 \%(\mathrm{P}<0.05)$ compared with control values.
And after 2.5-3.0 hours of the experiment, the average volumetric velocity index decreased to $1.0 \pm 0.3 \mu \mathrm{L} / \mathrm{min} \bullet \mathrm{g}$ of liver, i.e. by $10.3 \%$ compared with the control group of animals (Fig. 2). But Corvitin is effective on the composition of bile when used in conditions of chronic social stress. The concentration of taurocholic acid in bile when using Corvitin in the simulation of chronic social stress in male rats was higher than the control values from the first to the fifth sample by $1.4 \%, 7.1 \%, 9.2 \%(\mathrm{P}<0.05)$, $8.1 \%(\mathrm{P}<0.05)$ and $1.1 \%$. But in the sixth sample, the concentration of taurocholic acid was lower by $5.2 \%$ compared with the control (Table 1). The content of dihydroxycholate taurocholates in the experiment with Corvitin use in the simulation of chronic social stress was lower in all six samples by $12.1 \%, 10.3 \%, 9.0 \%, 7.2 \%, 12.2 \%$ and $14.7 \%(\mathrm{P}<0.05)$ respectively compared with the control values (Table 1). The content of glycocholates in bile when using Corvitin in the simulation of chronic social stress in male rats was higher than the control values from the first to the fifth sample by $21.9 \%, 31.7 \%(\mathrm{P}<0.05), 40.4 \%(\mathrm{P}<0.05), 30.4 \%$ $(\mathrm{P}<0.05)$ and $26.2 \%(\mathrm{P}<0.05)$, respectively. But in the sixth sample, the concentration of glycochenodeoxycholic and glycodeoxycholic acids was lower by $0.7 \%$ (Table 1$)$.

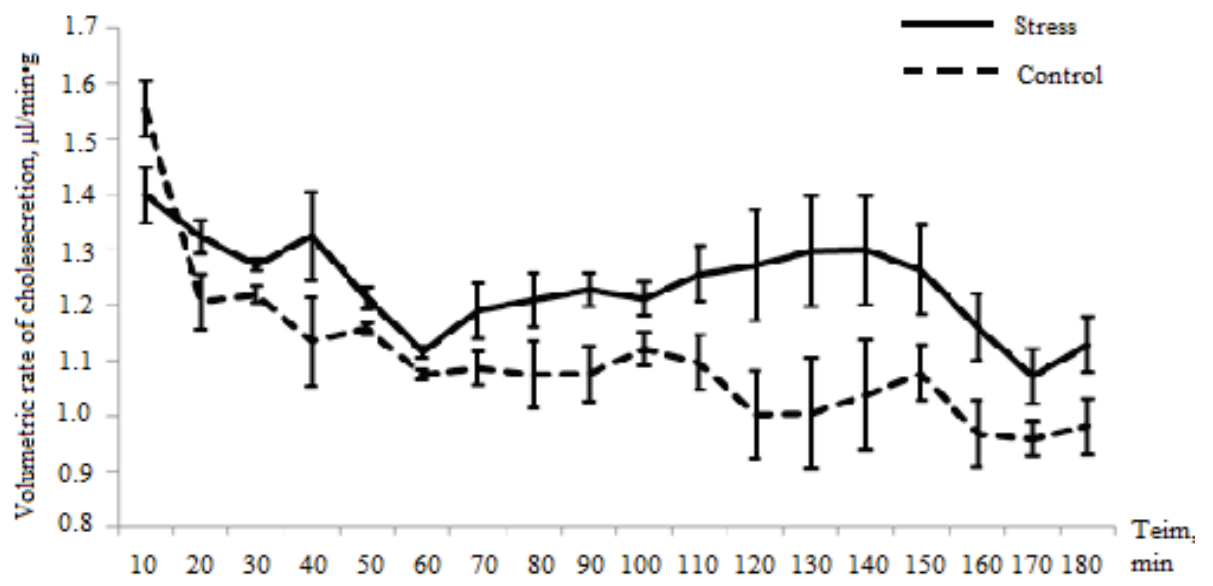

Fig. 1. Volumetric rate of cholesecretion one day after completion of the experimental social stress procedure in male rats $(\mathrm{x} \pm \mathrm{SD})$ : control $-\mathrm{n}=8$, stress $-\mathrm{n}=9$

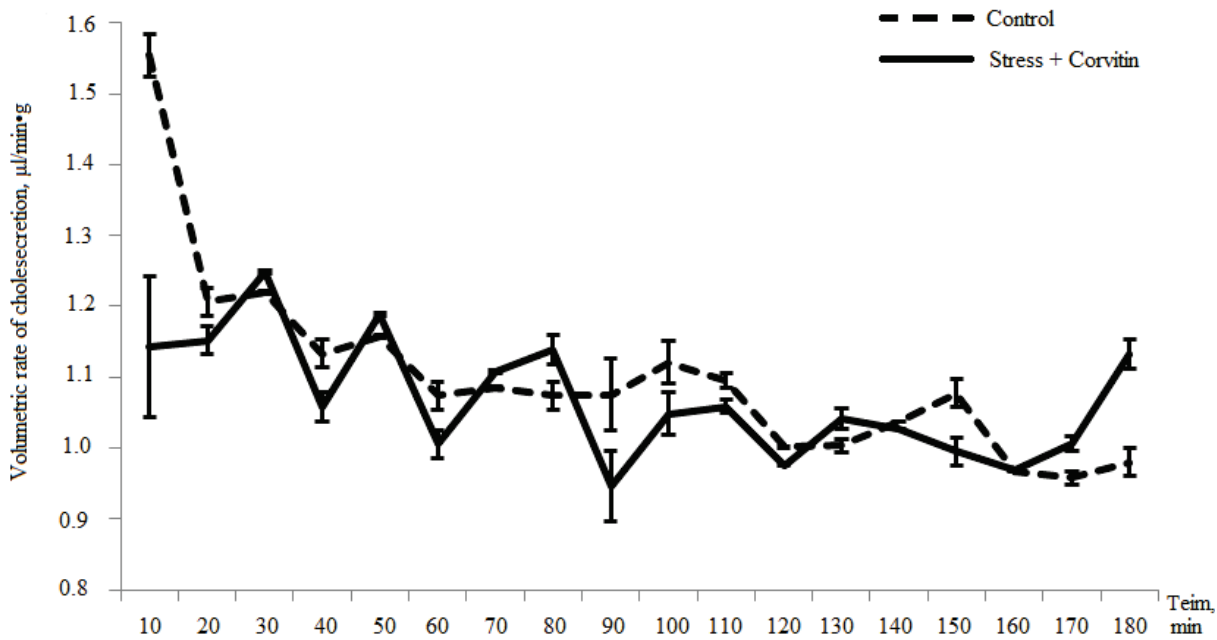

Fig. 2. Volumetric rate of cholesecretion when using Corvitin in the simulation of chronic social stress in male rats $(x \pm S D)$ : control $-\mathrm{n}=8$, stress + Corvitin $-\mathrm{n}=9$

Taurolithocholic and lithocholic acids appeared in the bile of stressed male rats when they were treated by Corvitin. In rats of the control group, neither taurolithocholate nor lithocholate was found (Table 1,2). In our studies, in the conditions of experimental social stress, Corvitin enhanced the conjugation of bile acids with taurine, i.e. stimulated detoxification processes in hepatocytes (Fig. 3).

Concentrations of cholic acid in the bile of male rats that received Corvitin in the conditions of modeling chronic social stress were lower by respectively $11.1 \%, 13.8 \%, 4.6 \%, 20.5 \%$ and $46.5 \%(\mathrm{P}<0.05)$ in all six samples, except the third, in comparison with the control values. In the third sample, the content of cholic acid was $0.2 \%$ higher than the control indicator (Table 2). The content of chenodeoxycholic and deoxycholic acid in bile when using Corvitin in the simulation of chronic social stress in male rats was higher than the control values from the first to the fifth sample by $22.1 \%, 30.3 \%, 26.8 \%, 16.7 \%$ and $3.9 \%$ respectively. However, in the sixth sample, the concentration of chenodeoxycholic and deoxycholic acid was lower than the control values by $24.9 \%(\mathrm{P}<0,05$; Table 2$)$.

Conjugation and hydroxylation indices were calculated and changes in these ratios were calculated to better evaluate the effect of Corvitin on bile acids in the liver of rats under chronic social stress and the characteris- 
tics of physicochemical properties of bile based on the concentration of cholates. The conjugation index allowed us to estimate the intensity of cholates binding with the amino acids taurine and glycine, which provides the ability to dissolve bile acids, and is important for the stability of bile as a colloidal solution and determines its ability to emulsify fats in the small intestine for further breakdown, their lipases of pancreatic and intestinal juices. In addition, the conjugation of cholates prevents their premature absorption in the proximal small intestine, which provides the concentration of bile acids required for the micellar phase of digestion and absorption of fats (Fuchs, 2003).

The conjugation index of bile acid of the bile when using Corvitin in the simulation of chronic social stress of male rats was $1.7 \%, 10.7 \%$ and $45.9 \%(\mathrm{P}<0.01)$ higher than the control values in the second, fifth and sixth samples. However, in the first, third and fourth samples, the bile acid of the bile conjugation rate was lower than the control values by $0.3 \%$, $2.7 \%$ and $1.8 \%$, respectively. Statistically significant differences in the bile acid conjugation rate of bile under the use of Corvitin in the simulation of chronic social stress in males compared with the control values were observed only in the sixth sample.

Analysis of changes in the hydroxylation index under the influence of Corvitin on the content of bile acids in the liver of rats under chronic social stress was performed to assess the activity of different pathways of bile acid biosynthesis - neutral (classical), whose products in humans are cholic and chenodeoxyzolic acid in approximately the same ratio and acidic (alternatively), which provides the formation of mainly chenodeoxyzolic acid (Kiriyama \& Nochi, 2019).

\section{Table 1}

Concentration of conjugated bile acids in the bile of control male rats $(n=8)$, with prolonged exposure to experimental chronic social stress $(n=9)$ and male rats after social stress when using Corvitin $(n=9, x \pm S D)$

\begin{tabular}{lccccc}
\hline \multicolumn{1}{c}{ Series of experiments } & $\begin{array}{c}\text { Taurocholic } \\
\text { acid, } 10^{-5}, \mathrm{~g} / \mathrm{L}\end{array}$ & $\begin{array}{c}\text { Taurohenodeoxycholic and } \\
\text { taurodeoxycholic acids, } 10^{-5}, \mathrm{~g} / \mathrm{L}\end{array}$ & $\begin{array}{c}\text { Taurolithocholic } \\
\text { acid, } 10^{-5}, \mathrm{~g} / \mathrm{L}\end{array}$ & $\begin{array}{c}\text { Glycocholic } \\
\text { acid, } 10^{-5}, \mathrm{~g} / \mathrm{L}\end{array}$ & $\begin{array}{c}\text { Glycohenodeoxycholic and } \\
\text { glycodeoxycholic acids, } 10^{-5}, \mathrm{~g} / \mathrm{L}\end{array}$ \\
\hline Control & $180.8 \pm 11.9$ & $103.1 \pm 8.3$ & 0 & $141.8 \pm 13.8$ & $23.6 \pm 6.2$ \\
Stress & $184.4 \pm 22.0$ & $100.9 \pm 11.6$ & $22.0 \pm 8.0^{* * *}$ & $95.1 \pm 34.7^{* * *}$ & $10.4 \pm 4.9^{* * *}$ \\
Stress + Corvitin & $183.3 \pm 13.4$ & $90.6 \pm 15.3$ & $4.2 \pm 5.2^{* *}$ & $139.7 \pm 14.2$ & $28.7 \pm 4.8$ \\
Control & $179.0 \pm 10.2$ & $104.5 \pm 8.5$ & 0 & $144.0 \pm 8.4$ & $21.9 \pm 4.5$ \\
Stress & $182.2 \pm 23.5$ & $100.9 \pm 12.1$ & $21.9 \pm 7.4^{* * *}$ & $91.3 \pm 32.6^{* * *}$ & $10.1 \pm 3.4^{* * *}$ \\
Stress + Corvitin & $191.8 \pm 14.1$ & $93.7 \pm 12.0$ & $3.2 \pm 4.3^{* *}$ & $138.7 \pm 10.6$ & $28.9 \pm 3.3^{*}$ \\
Control & $175.7 \pm 9.7$ & $99.8 \pm 8.5$ & 0 & $137.2 \pm 9.2$ & $20.8 \pm 5.0$ \\
Stress & $168.2 \pm 25.8$ & $91.9 \pm 10.0$ & $17.0 \pm 6.2^{* * *}$ & $82.5 \pm 29.4^{* * *}$ & $12.2 \pm 4.8^{* * *}$ \\
Stress + Corvitin & $191.8 \pm 11.0^{*}$ & $90.8 \pm 10.6$ & $2.2 \pm 2.8^{* *}$ & $140.5 \pm 7.6$ & $29.2 \pm 4.8^{*}$ \\
Control & $173.0 \pm 10.0$ & $95.9 \pm 10.4$ & 0 & $132.5 \pm 11.6$ & $20.4 \pm 4.2$ \\
Stress & $139.3 \pm 42.8$ & $77.7 \pm 19.4^{*}$ & $15.3 \pm 5.8^{* * *}$ & $72.8 \pm 26.5^{* * *}$ & $11.9 \pm 4.3^{* * *}$ \\
Stress + Corvitin & $187.0 \pm 5.4^{*}$ & $88.9 \pm 7.3$ & $0.9 \pm 1.2^{* *}$ & $127.3 \pm 7.4$ & $26.7 \pm 4.4^{*}$ \\
Control & $166.0 \pm 10.8$ & $92.8 \pm 9.6$ & 0 & $122.7 \pm 16.1$ & $19.1 \pm 4.1$ \\
Stress & $127.2 \pm 44,8^{*}$ & $74.7 \pm 16.0^{*}$ & $11.7 \pm 7.2^{* *}$ & $65.8 \pm 21.9^{* * *}$ & $12.6 \pm 5.0^{* *}$ \\
Stress + Corvitin & $167.9 \pm 12,7$ & $81.5 \pm 7.8$ & $0.4 \pm 2.2^{* *}$ & $111.6 \pm 6.8$ & $24.1 \pm 2.4^{*}$ \\
Control & $160.2 \pm 11,5$ & $89.8 \pm 8.0$ & 0 & $122.1 \pm 16.1$ & $17.4 \pm 3.7$ \\
Stress & $122.3 \pm 40.9^{*}$ & $69.0 \pm 13.8^{* * *}$ & $9.6 \pm 5.8^{* * *}$ & $59.5 \pm 19.2^{* * *}$ & $11.54 \pm 5.1^{*}$ \\
Stress + Corvitin & $150.7 \pm 11.5$ & $76.5 \pm 7.4^{*}$ & 0 & $89.5 \pm 12.7^{*}$ & $17.3 \pm 1.4$ \\
\hline
\end{tabular}

Notes: ${ }^{*}-\mathrm{P}<0.05,{ }^{* *}-\mathrm{P}<0.01,{ }^{* * *}-\mathrm{P}<0.001$ compared with the parameters of the control group with Bonferroni correction.

Table 2

The concentration of free bile acids in the bile of control male rats $(n=8)$, with prolonged exposure to experimental chronic social stress $(n=9)$ and male rats after social stress when using Corvitin $(n=9, x \pm S D)$

\begin{tabular}{|c|c|c|c|}
\hline Series of experiments & Cholic acid, $10^{-5}, \mathrm{~g} / \mathrm{L}$ & Chenodeoxycholic and deoxycholic acids, $10^{-5}, \mathrm{~g} / \mathrm{L}$ & Lithocholic acid, $10^{-5}, \mathrm{~g} / \mathrm{L}$ \\
\hline Control & $19.9 \pm 4.8$ & $8.3 \pm 2.0$ & 0 \\
\hline Stress & $13.1 \pm 2.6^{* *}$ & $9.5 \pm 1.5$ & $4.2 \pm 1.1^{* *}$ \\
\hline Stress + Corvitin & $17.7 \pm 4.3$ & $10.2 \pm 2.1$ & $0.2 \pm 0.2 *$ \\
\hline Control & $19.8 \pm 4.3$ & $7.9 \pm 1.3$ & 0 \\
\hline Stress & $13.2 \pm 2.6^{* *}$ & $9.9 \pm 1.6^{*}$ & $4.1 \pm 0.8^{* *}$ \\
\hline Stress + Corvitin & $17.1 \pm 3.1$ & $10.3 \pm 2.9$ & $0.2 \pm 0.2 *$ \\
\hline Control & $18.9 \pm 4.5$ & $7.5 \pm 1.2$ & 0 \\
\hline Stress & $12.2 \pm 1.4^{* *}$ & $9.0 \pm 1.4^{*}$ & $3.7 \pm 0.6^{* *}$ \\
\hline Stress + Corvitin & $18.9 \pm 3.9$ & $9.6 \pm 2.5$ & $0.3 \pm 0.3^{*}$ \\
\hline Control & $18.5 \pm 4.2$ & $7.4 \pm 1.1$ & 0 \\
\hline Stress & $10.5 \pm 2.2 * *$ & $8.0 \pm 1.4$ & $2.7 \pm 0.8^{* *}$ \\
\hline Stress + Corvitin & $17.6 \pm 2.7$ & $8.6 \pm 1.8$ & $0.4 \pm 0.4 *$ \\
\hline Control & $18.7 \pm 3.8$ & $7.4 \pm 0.9$ & 0 \\
\hline Stress & $10.5 \pm 2.2 * *$ & $7.6 \pm 1.7$ & $2.7 \pm 0.8^{* *}$ \\
\hline Stress + Corvitin & $14.9 \pm 3.0$ & $7.7 \pm 1.3$ & $0.5 \pm 0.5^{*}$ \\
\hline Control & $18.3 \pm 3.2$ & $7.4 \pm 0.8$ & 0 \\
\hline Stress & $9.5 \pm 1.8^{* *}$ & $7.2 \pm 1.5$ & $2.3 \pm 0.8^{* *}$ \\
\hline Stress + Corvitin & $9.8 \pm 1.5^{*}$ & $5.6 \pm 1.4^{*}$ & $0.2 \pm 0.3^{*}$ \\
\hline
\end{tabular}

Notes: ${ }^{*}-\mathrm{P}<0.05,{ }^{*}-\mathrm{P}<0.01$ compared with the parameters of the control group with Bonferroni correction.

The index of hydroxylation of bile acids bile when using Corvitin in the simulation of chronic social stress of male rats was higher than the control values during the first five samples by $3.9 \%, 2.7 \%, 2.3 \%, 1.9 \%$ and $0.4 \%$ respectively. In the sixth sample, there was $3.8 \%$ decrease in the hydroxylation index of bile acids compared with the control values. No statistically significant differences in the index of hydroxylation of bile acids under the use of Corvitin in the simulation of chronic social stress in male rats compared with the control values were observed. Compared with control values, the ratio of glycoconjugates and tauroconjugates of bile acids in bile of male rats when using Corvitin in the simulation of chronic social stress was $6.9 \%$ and $3.5 \%$ higher in the first and third samples. At minutes 120 and 180 of the acute experiment, there was decrease in the ratio of glycoconjugates and tauroconjugates of bile acids in bile from the control values by $1.8 \%$ and $14.6 \%$, respectively. There were no statistically 
significant differences in the ratio of glycoconjugates and tauroconjugates of bile acids in bile when using Corvitin in the simulation of chronic social stress in male rats compared to the control values.

\section{Discussion}

The formation and secretion of bile is a specific function of the liver, which depends on the course of numerous metabolic reactions in its cells. Bile production is a vital process, which is provided by many external and internal factors. The formation of bile in hepatocytes is due to the intensity of metabolic reactions, the result of which, firstly, is the synthesis and biotransformation of organic components of bile and many enzymes and transport systems of the cell, and secondly, energy supply of organic and inorganic components of bile from blood to hepatocytes, intracellular transport and secretion of these components across the apical membrane to the primary bile ducts (Boyer, 2008).

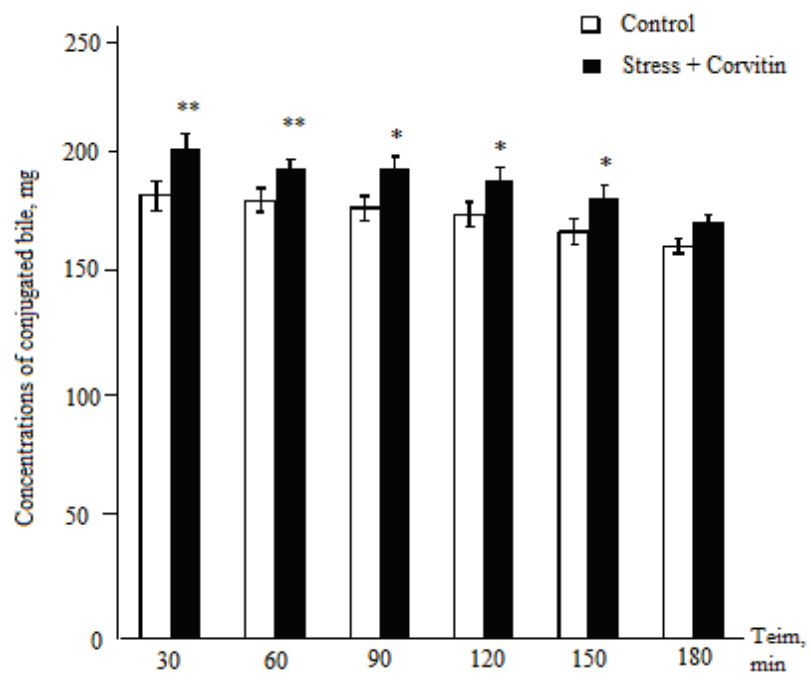

Fig. 3. Concentrations of conjugated bile (taurocholic) in bile when using Corvitin in the simulation of social stress of male rats: control $(\mathrm{n}=8)$, stress + Corvitin $(\mathrm{n}=9, \mathrm{x} \pm \mathrm{SD}) ;{ }^{*}-\mathrm{P}<0.05$, $* *-\mathrm{P}<0.01$ compared with the parameters of the control group with Bonferroni correction

Bile acids are endogenous regulators of certain intracellular metabolic processes, activation or inhibition of which leads to changes in the intensity of bile secretion and its organic components. The studies revealed a relationship between choleretic effects of bile acids and changes in protein biosynthesis in hepatocytes (Arab et al., 2017). It is also assumed that their effect on bile secretion is due to their effects on metabolic processes that form the basis of bile secretory function. This presumption is supported by data on the activation of the processes of synthesis, biotransformation and conjugation of bile acids in hepatocytes, multidirectional changes in RNA polymerase activity of isolated hepatocyte nuclei under the influence of cholic (suppression) and taurocholic (stimulation) acids, dependence of bile bicarbonate (Marin et al., 2017).

Substances that significantly affect liver function include bioflavonoids, including quercetin. Quercetin is used in the treatment of liver disease. It improves the biomarkers of liver tissue after various damages. It was found in the experiment that quercetin derivatives can cause changes in bile secretory function (Vovkun et al., 2016). We studied the effect of the water-soluble form - Corvitin on the spectrum of bile acids in the bile of rats under conditions of modeling chronic social stress.

Studies have shown that under conditions of experimental social stress Corvitin enhances the conjugation of bile acids with taurine and glycine, i.e. stimulates detoxification processes in hepatocytes. Taurolithocholic acid was found in the bile of stressed male rats treated with Corvitin. Thus, the use of Corvitin did not prevent the appearance of secondary bile acid - lithocholate in the liver secretion. Lithocholic acid belongs to the so-called secondary bile acids and has much more pronounced detergent and, consequently, damaging properties than di- and trihydroxycholan primary bile acids (Matsubara et al., 2011). In the normal conditions it is excreted from the body, or hydroxylates in hepatocytes. In rats that were not subjected to stress, neither taurolithocholate nor lithocholate acids were found. The concentration of conjugated cholates in the liver secretion of stressed male rats was lower than in control animals. Even when using Corvitin under chronic social stress, these figures did not return to normal.

In male rats suffering chronic social stress, the processes that provided the flow of glycoconjugates of bile acids from hepatocytes to the bile ducts were further suppressed. The concentrations of glycocholic acid and glycochenodeoxycholic and glycodeoxycholic acids in the bile of male intruders were lower than the control values (Liashevych et al., 2017). The use of Corvitin in the conditions of experimental social stress normalized these indicators.

Cholic acid belongs to the so-called primary bile acids, which are directly synthesized by the enzymatic systems of hepatocytes from cholesterol (Boyer, 2013; Chiang, 2013). Its concentration reflects the intensity of synthetic energy-dependent processes in liver cells (Liashevych et al., 2017). The studies demonstrated that in the conditions of chronic social stress, the content of cholic acid in the bile of intruder rats decreased, and when correcting the pathological condition using Corvitin, it approached the control values.

The conjugation index of bile acids of the bile is one of the integrative indicators of the coherence of the systems functioning of the metabolic transformations and cholates transport, primarily in hepatocytes. In general, an indicator such as the bile acid of the bile conjugation ratio provides information on the solubilization properties of bile, and the ratio of free and conjugated cholate fractions is one of the criteria for assessing the bile lithogenicity. Disruption of bile acid biosynthesis, including their conjugation, for example due to congenital metabolic defects, leads to serious consequences for health and life (Clayton, 2011; Setchell et al., 2013). Conjugation rate of the bile acids of bile in the context of using Corvitin during the simulation of chronic social stress in male rats was the highest in the sixth sample, greater by $45.9 \%(\mathrm{P}<0.01)$ compared with the control.

Conjugation of free cholate with taurine and glycine - the last stage in the biosynthesis of primary and biotransformation of secondary bile acids and its physiological content provides a higher micellar concentration of cholate in the intestinal cavity, which, in turn, improves the absorption of lipids and fat-soluble vitamins (Clayton, 2011). Pathological disorders of formation of taurine-glycine-conjugated cholates cause malabsorption of fat-soluble vitamins and cholestatic liver damage (Heubi et al., 2013).

\section{Conclusions}

Studies of the effect of Corvitin on bile acid content in the liver of rats under chronic social stress allowed us to determine significant differences between the indicators in male intruder rats compared with control animals. The transport of bile acids to the bile was disrupted in male ratsintruders, who were exposed to chronic social stress. As a result, the concentration of free cholic acid and glycocholates in the bile of stressed animals decreased. Social stress caused changes in bile production, which decreased the solubilization properties of bile and increased the risk of lithogenesis after the end of exposure to chronic stress.

The use of Corvitin simultaneously with the simulation of experimental social stress normalizes the biliary function of the liver, which indicates the high potential of Corvitin as a corrective factor in chronic social stress. Corvitin used in the conditions of experimental social stress has to some extent corrected the content of bile acids in the liver of male rats, which indicates the ability of this drug to interfere with the metabolism of cholate in liver cells in the mechanisms of bile acid transport.

Thus, chronic social stress leads to certain changes in the ratio of lipids and bile acids in the bile, which must be taken into account when correcting stress-induced pathologies. Correction of stress-induced pathologies of bile-secretory function of the liver using Corvitin requires further thorough experimental studies.

\section{References}

Arab, J. P., Cabrera, D., \& Arrese, M. (2017). Bile acids in cholestasis and its treatment. Annals of Hepatology, 16, 53-57.

Boyer, J. L. (2008). Bile canalicular secretion - tales from Vienna and Yale. Wiener Medizinische Wochenschrift, 158(19), 534-538. 
Boyer, J. L. (2013). Bile formation and secretion. Journal of Comparative Physiology, 3(3), 1035-1078.

Brygadyrenko, V. V., Lieshchova, M. A., Bilan, M. V., Tishkina, N. M., \& Horchanok, A. V. (2019). Effect of alcohol tincture of Aralia elata on the organism of rats and their gut microbiota against the background of excessive fat diet. Regulatory Mechanisms in Biosystems, 10(4), 497-506.

Chiang, J. Y. (2013). Bile acid metabolism and signaling. Joumal of Comparative Physiology, 3(3), 1191-1212.

Chiang, J. Y. (2017). Bile acid metabolism and signaling in liver disease and therapy. Liver Research,1(1), 3-9.

Chuang, J. C., Cui, H., Mason, B. L., Mahgoub, M., Bookout, A. L., Hana, G. Y., Perello, M., Elmquist, J. K., Repa, J. J., Zigman, J. M., \& Lutter, M. (2010). Chronic social defeat stress disrupts regulation of lipid synthesis. Journal of Lipid Research, 51(6), 1344-1353.

Clayton, P. T. (2011). Disorders of bile acid synthesis. Journal of Inherited Metabolic Disease, 34(3), 593-604.

Fuchs, C., Claudel, T., \& Trauner, M. (2013). Bile acid-mediated control of liver triglycerides. Seminars in Liver Disease, 33(4), 330-342.

Fuchs, M. (2003). Bile acid regulation of hepatic physiology: III Regulation of bile acid synthesis: past progress and future challenges. The American Journal of Physiology-Gastrointestinal and Liver Physiology, 284(4), 551-557.

Giudetti, A. M., Testini, M., Vergara, D., Priore, P., Damiano, F., Gallelli, C. A., Romano, A., Villani, R., Cassano, T., Siculella, L., Gnoni, G. V., Moles, A., Coccurello, R., \& Gaetani, S. (2019). Chronic psychosocial defeat differently affects lipid metabolism in liver and white adipose tissue and induces hepatic oxidative stress in mice fed a high-fat diet. The FASEB Joumal, 33(1), 1428-1439.

Heubi, J. E., Setchell, D. R., Jha, P., Buckley, D., Zhang, W., Rosenthal, P., Potter, C., Horslen, S., \& Suskind, D. (2015). Treatment of bile acid amidation defects with glycocholic acid. Hepatology, 61(1), 268-274.

Horid'ko, T. M., Kosiakova, H. V., Berdyshev, A. G., Meged, O. F., Gudz, E. A., Onopchenko, O. V., Asmolkova, V. S., Lozova, V. M., Tukalenko, E. V., Bondarenko, O. V., Tubalzeva, I. I., Kovalenko, O. A., Makarchuk, M. Y., \& Hula, N. M. (2017). Antistress effects of n-stearoylethanolamine in rats with chronic social stress. The Ukrainian Biochemical Journal, 89(4), 68-76.

Jensen, M. P., Sherlin, L. H., Askew, R. L., Fregni, F., Witkop. G., Gianas, A., \& Hakimian, S. (2016). Effects of non-pharmacological pain treatments on brain states. Clinical Neurophysiology, 124, 2016-2024.

Juza, R. M., \& Pauli E. M. (2014). Clinical and surgical anatomy of the liver: A review for clinicians. Clinical Anatomy, 27(5), 764-769.

Kiriyama, Y, \& Nochi, H. (2019). The biosynthesis, signaling, and neurological functions of bile acids. Biomolecules, 9(6), 232.

Kudryavtseva, N. (1991). A sensory contact model for the study of aggressive and submissive behavior in male mice. Aggressive Behavior, 17(5), 285-291.

Liashevych, A. M., Tubalceva. I. I., Reshetnik, Y. M., Bondarenko, O. V., Veselsky, S. P., \& Makarchuk, M. Y. (2017). Influence of experimental chronic social stress on bile acids content in the bile of male rats. Fiziologichnyi Zhurnal, 63(4), 24-29.

Lieshchova, M. A., Tishkina, N. M., Bohomaz, A. A., Gavrilin, P. M., \& Brygadyrenko, V. V. (2018). Combined effect of glyphosphate, saccharin and sodium benzoate on rats. Regulatory Mechanisms in Biosystems, 9(4), 591-597.
Maleszka, A., Dumnicka, P., \& Matuszyk, A. (2017). The diagnostic usefulness of serum total bile acid concentrations in the early phase of acute pancreatitis of varied etiologies. International Journal of Molecular Sciences, 18(1), 106.

Marin, J. G., Macias, I. R., Briz, O., Banales, J. M., \& Monte, M. J. (2015). Bile acids in physiology, pathology and pharmacology. Current Drug Metabolism, 17(1), 4-29.

Matsubara, T., Tanaka, N., Patterson, A. D., Cho, J. Y., Krausz, K. W., \& Gonzalez, F. J. (2011). Lithocholic acid disrupts phospholipid and sphingolipid homeostasis leading to cholestasis. Hepatology, 53(4), 1282-1293.

Pierre, J. F., Martinez, K. B., Honggang, Y., Nadimpalli, A., Morton, T. C., Yang, J., Wang, Q., Patno, N., Chang, E. B., \& Yin, D. P. (2016). Activation of bile acid signaling improves metabolic phenotypes in high-fat diet-induced obese mice. The American Journal of Physiology: Gastrointestinal and Liver Physiology, 311(2), 286-304.

Scott, K. A, Melhorn. S. J., \& Sakai, R. R. (2012). Effects of chronic social stress on obesity. Current Obesity Reports, 1(1), 16-25.

Setchell, D. R., Heubi, J. E., Shah, S., Lavine, J. E., Suskind, D., Al-Edreesi, M., Potter, C., Russell, D. W., O'Connell, N. C., Wolfe, B., Jha, P., Zhang, W., Bove, K. E., Knisely, A. S., Hofmann, A. F., Rosenthal, P., \& Bull, L. N. (2013). Genetic defects in bile acid conjugation cause fat-soluble vitamin deficiency. Gastroenterology, 144(5), 945-955.

Shkurashivska, S. V., \& Ersteniuk, H. M. (2015). Dynamics of lipid metabolism in tissues and organs of experimental animals under conditions of stress adrenaline. Clinical Chemistry and Laboratory Medicine, 17(4), 34-37.

Silvennoinen, R., Quesada, H., Kareinen, I., Julve, J., Kaipiainen, L., Gylling, H., Blanco-Vaca, F., Escola-Gil, J. C., Kovanen, P. T, \& Lee-Rueckert, M. (2015). Chronic intermittent psychological stress promotes macrophage reverse cholesterol transport by impairing bile acid absorption in mice. Physiological Reports, 3(5), 12-14.

Taoka, H., Yokoyama, Y., Morimoto, K., Kitamura, N., Tanigaki, T., Takashina, Y., Tsubota, K., \& Watanabe, M. (2016). Role of bile acids in the regulation of the metabolic pathways. World Journal of Diabetes, 7(13), 260-270.

Trauner, M., Claudel, T., Fickert, P, Moustafa, T., \& Wagner, M. (2010). Bile acids as regulators of hepatic lipid and glucose metabolism. Digestive Diseases, 28(1), $220-224$.

Vasheka, I. P., Veselskyi, S. P., Horenko, Z. A., Hrinchenko, O. A., Karbovska, L. S., \& Makarchuk, M. Y. (2014). Impact on amilinu range holativ bile in rats. Fiziologichnyi Zhurnal, 60(3), 46-53.

Vovkun, T. V., Yanchuk, P. I., Shtanova, L. Y., Veselskyy, S. P., \& Shalamay, A. S. (2016). Exocrine function of the liver in rats with exposure to corvitin. Fiziologichnyi Zhumal, 62(3), 30-38.

Yan, L., \& Lun-Gen, L. (2018). Therapeutic roles of bile acid signaling in chronic liver diseases. Journal of Clinical and Translational Hepatology, 6(4), 425-430.

Yun-Zi, L., Wei, P., Ji-Kuai, C., Wen-Jun, S., Wen-Jie, Y., Yun-Xia, W., \& ChunLei, J. (2019). FoxO1 is a critical regulator of hepatocyte lipid deposition in chronic stress mice. PeerJ, 7, 76-78.

Zhao, C., \& Dahlman-Wright K. (2010). Liver X receptor in cholesterol metabolism. Journal of Endocrinology, 204(3), 233-240.

Zhou, H., \& Hylemon, P. B. (2014). Bile acids are nutrient signaling hormones. Steroids, 86, 626-628. 